\title{
Diseños de Aprendizaje Basados en las TIC (Moodle 2.0 y Mahara) para Contenidos de Anatomía, Fisiología y Salud en las Clases de Educación Física Escolar
}

\author{
Patterns of ICT-Based Learning (Moodle and Mahara 2.0) for Contents of \\ Anatomy, Physiology and Health in Scholar Physical Education Lessons
}

\author{
Antonio Granero-Gallegos* \& Antonio Baena-Extremera**
}

GRANERO-GALLEGOS, A. \& BAENA-EXTREMERA, A. Diseños de aprendizaje basados en las TIC (Moodle 2.0 y Mahara) para contenidos de Anatomía, Fisiología y Salud en las clases de Educación Física escolar. Int. J. Morphol., 33(1):375-381, 2015.

RESUMEN: El objetivo fue comparar el rendimiento en el aprendizaje de alumnado de secundaria en clase de Educación Física tras recibir dos unidades didácticas en las que la formación teórica de los contenidos de Anatomía, Fisiología y Salud Corporal, fue impartida con el apoyo de Moodle 2.0 y Mahara. Se utilizó un diseño cuasi-experimental, descriptivo y seccional. La muestra total se compuso por 122 alumnos de $4^{\circ}$ de Enseñanza Secundaria Obligatoria (ESO), 57 varones y 65 mujeres. Se trabajó con un grupo experimental de 72 personas que recibieron la formación teórica de dos unidades didácticas con apoyo de Moodle 2.0 y Mahara, y otro grupo control de 50 alumnos que recibió las clases sin apoyo de plataformas virtuales. Las clases prácticas fueron igual en ambos grupos. El instrumento de medida fue un cuestionario de evaluación tipo test. Se obtuvo una medida pretest antes de la formación y otra medida después de la intervención (postest). Se han analizado las mejoras de cada grupo según el método de enseñanza teórica empleado y se han comparado los resultados obtenidos mediante análisis de la puntuación de ganancia (postest-pretest) con T de Student para muestras relacionadas. Tras la intervención, la media de respuestas correctas es superior al cincuenta por ciento en ambos grupos, siendo mayores las puntuaciones de ganancia de fisiología, anatomía y salud en el grupo experimental. Estos datos corroboran que la utilización de las plataformas LMS contribuyen en mayor medida a la adquisición de estos contenidos entre el alumnado. Una importante aportación en el campo de la EF lo constituye el hecho de que sean necesarias menos horas presenciales de carácter teórico, pues el alumnado trabaja en horario extralectivo sobre los contenidos prácticos realizados en clase, disponiendo así de más horas de práctica lectiva.

PALABRAS CLAVE: Metodología; Moodle 2.0; Mahara; Anatomía; Fisiología; Educación Física.

\section{INTRODUCCIÓN}

La enseñanza y aprendizaje de los contenidos de diferentes materias, tanto de la enseñanza obligatoria de primaria como en la post-obligatoria de secundaria y universitaria, están sufriendo profundos procesos de cambio al incluir dentro de sus metodologías de clase las nuevas tecnologías de la información y comunicación (TIC). Hoy día apenas se manifiestan dudas en relación a la importancia que las TIC están teniendo en el mundo de la educación (Gutiérrez et al., 2010; Suárez-Rodríguez et al., 2013); tanto es así que los docentes, para que sus conocimientos no se queden anticuados y poder dar respuesta a las necesidades de la sociedad actual, se ven obligados a actualizarse y participar en actividades de formación continua relacionadas con las innovaciones tecnológicas (Padilla-Meléndez et al., 2015).
El hecho de que exista un avance tecnológico y éste se incluya en el sistema educativo no es obligatoriamente síntoma de una mejora en la enseñanza-aprendizaje del alumnado (ver por ejemplo el trabajo presentado por BaenaExtremera \& Granero-Gallegos (2013). Autores como Correa \& de Pablos (2009) creen que la introducción de las tecnologías en los centros educativos no obedece a intereses educativos, sino económicos, por lo que se estaría produciendo una separación del verdadero sentido de la utilización didáctica de estos recursos en los centros educativos. De hecho, autores como Rodríguez (2010) manifiestan que la integración de las TIC en modelos formativos no adecuados no solo no mejora el aprendizaje sino que lo empeora, incrementando la carga a profesorado y estudiantes. A pesar de ello, diversos autores, como Ramos \& Martínez (2011),

\footnotetext{
* Centro del Profesorado Almería 03. Consejería de Educación. Junta de Andalucía, Andalucía, España.

*** Facultad de Ciencias del Deporte. Universidad de Murcia, Murcia, España.
} 
se manifiestan a favor de su inclusión, pues han podido constatar mejoras en los discentes.

Agüera \& de la Haba (2009) afirman que la utilización de las TIC, en especial en las ciencias, puede ser una herramienta complementaria de apoyo a la formación práctica de los alumnos. Según estos autores, el uso de las TIC, no sólo en las clases teóricas sino en los trabajos de laboratorio, constituyen un recurso didáctico fundamental en la enseñanza, ya que permite al alumno comprobar el grado de asimilación de los contenidos teóricos.

Algieri et al. (2009) utilizan las TIC en la enseñanza del sistema digestivo, mientras que Bucarey et al., (2011) proponen diseños de aprendizaje de contenidos anatómicos basados en LAMS e integrado en Moodle. Otros autores como Prieto et al. (2011) utilizan un blog en la comparación de la placenta, obteniendo resultados muy satisfactorios. Incluso Granero-Gallegos (2008) expone el éxito de la utilización de la plataforma Moodle en una experiencia de teleformación con profesorado de Educación Física (EF) (primaria y secundaria) en actividades físicas en el medio natural. Rodríguez cree que la utilización de las TIC en la educación a distancia y en el apoyo on-line a la educación presencial está creciendo notablemente. De ahí, que gran parte de los recursos utilizados sean la plataforma Moodle 2.0 y Mahara, por su interés y funcionalidad.

Moodle consiste en un software o entorno virtual del tipo Plataforma de Gestión del Aprendizaje (LMS, Learning Management System) para desarrollar cursos a distancia, que permite crear comunidades de aprendizaje en línea y que facilita la comunicación entre profesores y alumnos (Pérez, 2006). Según Betegón et al. (2010), Moodle, significa Entorno de Aprendizaje Dinámico Orientado a Objetos y Modular (Modular Object-Oriented Dynamic Learning Environment), y se define no sólo como un sistema de gestión de cursos (un paquete de programa diseñado para ayudar al profesor a crear fácilmente cursos de calidad en línea), sino como un medio de aprendizaje capaz de estimular al alumno. En relación a Mahara, indicar que es un portafolio con todas las funciones de código abierto electrónico, en el que se pueden crear sistema de redes sociales, conectar a los usuarios entre ellos y crear comunidades en línea.

Es aún escasa la utilización de estas plataformas en el proceso de enseñanza de contenidos anatómico-fisiológicos y de salud humana. Por ello, una vez contextualizado este proyecto, se planteó trabajar contenidos de Anatomía, Fisiología y Salud con alumnado de secundaria a través de dos metodologías diferentes, tradicional y con TIC. El objetivo de este estudio fue comparar el rendimiento en el aprendizaje de alumnado de secundaria en clase de Educación Física, tras recibir dos unidades didácticas en las que la formación teórica de los contenidos de Anatomía, Fisiología y Salud Corporal, fue impartida con apoyo de Moodle 2.0 y Mahara.

\section{MATERIAL Y MÉTODO}

Muestra. En esta investigación participaron un total de 122 alumnos en clases de EF pertenecientes al $4^{\circ}$ curso de Educación Secundaria Obligatoria (ESO), con edades comprendidas entre 15 y 16 años $(M=15,23$; $D T=0,65)$. Entre el alumnado, 57 eran chicos y 65 eran chicas, pertenecientes a un centro de enseñanza pública de la provincia de Almería (España). Las clases eran mixtas y los contenidos impartidos en el grupo experimental y en el grupo control fueron los mismos, aunque la metodología empleada fue diferente.

Diseño. Se utilizó un diseño cuasi-experimental, descriptivo y seccional. Se trabajó con grupo control no equivalente, puesto que los grupos no fueron asignados al azar, al estar previamente establecidos por el centro educativo. Participaron cinco grupos de $4^{\circ}$ de ESO. Tras sorteo -elección al azar- los tres grupos designados por el centro educativo con las letras B, D y E fueron experimentales y estaban compuestos por 25, 24 y 23 alumnos, respectivamente, formando un total de 72 personas. Estos grupos experimentales recibieron clase de los contenidos teóricos de las unidades didácticas a través de Moodle 2.0 y Mahara como apoyo para el desarrollo de los contenidos prácticos: foros, links, wikis, vídeos, presentaciones, actividades condicionadas, cuestionarios, etc. Asimismo, mediante el uso de las TIC, el docente pudo tener en todo momento mayor control sobre el proceso de aprendizaje del alumnado. Para evitar posibles interferencias en los resultados y amenazas en los efectos de la muestra, los alumnos no supieron en ningún momento si formaban parte del grupo experimental o control.

Los grupos designados por el centro como A y C constituyeron los dos grupos control y estaban compuestos por 24 y 26 alumnos, respectivamente, formando un total de 50 personas. Estos grupos control recibieron las clases prácticas de igual manera que los grupos experimentales, pero sin apoyo de las expuestas plataformas virtuales para los contenidos teóricos; éstos fueron impartidos siguiendo metodologías tradicionales (clase magistral, pizarra y apuntes dictados de forma oral, fotocopias, etc.). Todas las clases tuvieron la misma duración para todos los grupos y se trataron los mismos contenidos, pero diferenciando los enfoques metodológicos indicados.

Se realizó una primera medida teórica (evaluación 
inicial de la unidad didáctica) a todos los grupos (pretest) para conocer el nivel inicial de conocimientos relacionados con contenidos anatómicos, fisiológicos y de salud. Esta medida pretest se realizó antes de iniciar el período en el que se tratarían los contenidos seleccionados. Este pretest se utilizó también para evaluar la normalidad de la muestra, siendo desestimadas 2 personas en el grupo B, 2 en el grupo D, por demostrar conocimientos fuera de la normalidad. Una vez finalizado el proceso enseñanza-aprendizaje de las dos unidades didácticas, se realizó otra prueba postets a todos los grupos con el objeto de establecer comparaciones con el pretest.

Hipótesis. Respecto a la hipótesis planteada para esta investigación, se piensa que a partir de la intervención se producirán mayores diferencias significativas de medias y en la puntuación de ganancia en el postest entre los sujetos del grupo experimental y los del grupo control (postest intergrupos).

Instrumento de medida. Para llevar a cabo las evaluaciones de los conocimientos se diseñó un cuestionario final que integraba preguntas referentes a las unidades didácticas tratadas. El cuestionario estaba compuesto por 50 preguntas tipo test, de selección múltiple y con una sola respuesta válida. Del total de cuestiones, 30 hacían referencia clara a contenidos anatómicos, fisiológicos y de salud (10 a cada bloque), y los otros 20 ítems se referían a otro tipo de contenidos relacionados con las unidades de trabajo. Todas las pruebas escritas se prepararon antes de la intervención por el equipo de profesorado e investigadores con el fin de evitar cualquier tipo de sesgo en la preparación de las pruebas tras la intervención. Las cuestiones referentes a los diferentes contenidos se intercalaron entre las demás cuestiones y hacían referencia tanto a aspectos conceptuales, como a aspectos de aplicación en la práctica física. Tanto en el pretest como en el postest se dispuso de 50 minutos para dar respuesta a todas las cuestiones. El cuestionario se pasó el mismo día a todo el alumnado, sin aviso previo.

\section{Características del programa y contenidos de anatomía, fisiología y salud}

Las sesiones se llevaron a cabo entre los meses de septiembre a noviembre de 2011. El centro educativo elegido contaba con la infraestructura suficiente para poder desarrollar los contenidos de las unidades didácticas, además de la infraestructura tecnológica adecuada para el desarrollo de sesiones con TIC.

Los contenidos estaban distribuidos en dos unidades didácticas pertenecientes al bloque de contenidos de Condición Física y Salud. En la primera unidad didáctica se trabajaron los contenidos de Anatomía y Fisiología y tuvo una duración de 4 semanas, con un total de 8 horas de clase. De estas 8 horas el grupo control recibió 3 sesiones teóricas y las otras 5 fueron prácticas; mientras que el grupo experimental recibió solamente 1 sesión teórica (la primera; para explicar la forma en que se iba a llevar a cabo el trabajo teórico y dar claves de acceso a las plataformas). En la segunda unidad didáctica se trataron contenidos de Fisiología y Salud, con una duración de 5 semanas y con un total de 10 horas de clase: el grupo control recibió 3 sesiones teóricas, mientras que el grupo experimental solamente recibió 1. Todas las dudas y consultas que surgieron de los componentes del grupo experimental se resolvieron en el espacio virtual.

Además de las clases teóricas durante el horario lectivo, para trabajar los contenidos en horario extralectivo, al grupo control se les encargó trabajo de carácter extraescolar (fichas y trabajos escritos según la metodología tradicional). El grupo experimental tuvo acceso a los contenidos teóricos y trabajos a través de la Moodle y Mahara.

A partir del Real Decreto 1631/2006 de 29 de diciembre, por el que se establecen las enseñanzas mínimas para la Educación Secundaria Obligatoria en España, para el área de EF el equipo docente ha decidido trabajar en $4^{\circ}$ curso (Agencia Estatal Boletín Oficial del Estado, 2006), con los contenidos que se observan el la Figura 1.

Procedimiento. Para evitar la amenaza por efecto del experimentador, todos los grupos recibieron las sesiones en el aula de EF por el mismo profesor (docente habitual y participante en este proyecto) durante el periodo de la investigación y siguiendo con total normalidad la programación de la asignatura. La medida pretest se realizó en la primera clase teórica, antes de comenzar las unidades de trabajo; los postest se realizaron una semana después de terminar las unidades didácticas con la metodología utilizada (Fig. 2). De esta manera, corregimos las limitaciones aportadas por Alleyne et al. (2002) y Jones et al. (2002), en estudios de este tipo. Fueron descartados los cuestionarios de aquel alumnado que no asistió al menos a ocho sesiones durante el periodo en cuestión. Esto supuso la pérdida en la medida postest de 4 sujetos en el grupo experimental y 2 en el grupo control.

Análisis. Las respuestas correctas fueron codificadas con el número 1 y las incorrectas con el 0 para el análisis estadístico. En cada uno de los bloques de cuestiones se calcularon los estadísticos descriptivos, media (M) y desviación típica (DT). Se realizaron las oportunas pruebas de normalidad y homocedasticidad para asegurar la homogeneidad de la varianza. Se han calculado los índices de asimetría y curtosis resultando, en general, próximos a cero y $<2,0$, como recomiendan Bollen \& Long (1994). De forma preliminar se realizó una evaluación inicial y se tuvo en cuenta el cómputo 


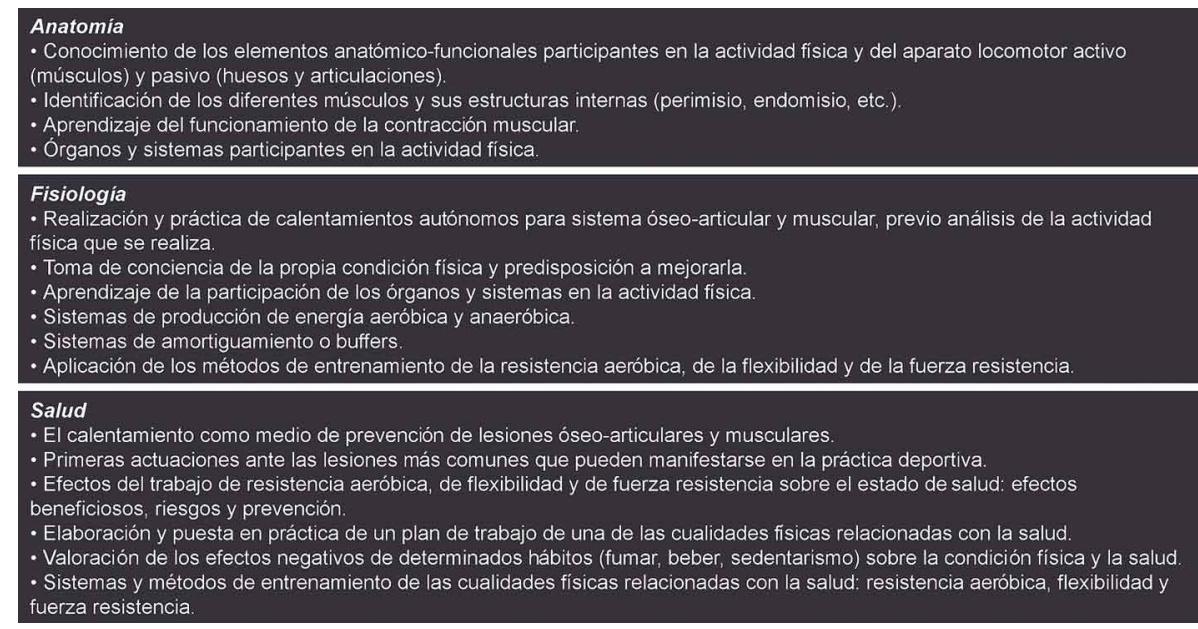

Fig. 1. Bloques temáticos y contenidos.

de respuestas correctas del grupo experimental y del grupo control en cada uno de los contenidos. Tras el programa de intervención, y con el objeto de estudiar las diferencias intragrupo en las diferentes tomas (pre y postest), se realizó un análisis de las puntuaciones de diferencia o análisis de puntuación de ganancia (Best, 1970). Se ha calculado la diferencia entre las puntuaciones postest y pretest para cada persona, analizando a continuación las diferencias intragrupo mediante la prueba $T$ de Student para muestras relacionadas. Para todo ello se empleó SPSS v. 17.0.

\section{RESULTADOS}

Análisis preliminar. En primer lugar se analizaron las diferencias inter-grupos mediante la Prueba $T$ para muestras independientes con el objeto de comprobar si se partía de dos grupos homogéneos. Los estadísticos más relevantes de las medidas pretest se recogen en la Tabla I. Los resultados mostraron que no existían diferencias significativas en ninguna de los bloques de contenidos analizados, tratándose, pues, de grupos homogéneos de partida: anatomía $\left(\mathrm{F}_{122}=2,03 ; t=-0,288\right.$; $\mathrm{p}=0,774)$, fisiología $\left(\mathrm{F}_{122}=0,001 ; t=-0,565 ; \mathrm{p}=0.573\right)$, salud $\left(\mathrm{F}_{119,306}=4,08 ; t=0,356, \mathrm{p}=0,722\right)$. Se comprueba que en ninguno de los casos la media alcanza el cincuenta por ciento de respuestas correctas. El contenido en el que se obtiene mejor puntuación es el de salud $(M=4,30)$, seguido de anatomía $(\mathrm{M}=2,78) \mathrm{y}$, por último, el de fisiología, en el que apenas se logra una media de 1,23 respuestas correctas.

Efectos de la intervención. Pasada una semana de la finalización de la intervención se realizó una medida postest con 10 cuestiones para cada uno de los bloques de contenidos. En la Tabla I se expone la evolución general del total de la muestra en los diferentes bloques. Después del programa la media de respuestas correctas es superior al sesenta y siete por ciento en los tres contenidos. Los mejores resultados los presentan salud $(M=7,30)$ y anatomía $(M=7,22)$, seguidos por los de fisiología $(M=6,78)$.

No obstante, expuesto lo anterior, se ha de llamar la atención acerca de los contenidos en los que más han evolucionado los alumnos tras la metodología empleada. Con el objeto de conocer qué método de enseñanza-aprendizaje resultó más efectivo se ha estudiado la puntuación de ganancia mediante la Prueba $T$ de Student para muestras relacionadas (diferencias intragrupo). En la Tabla II se pueden observar la M, DT y puntuación de ganancia de cada uno de los grupos, tanto en el pretest como en el postest.

Tras el proceso de intervención se han producido mejoras significativas tanto en el grupo experimental como en el grupo control $(\mathrm{p}<, 000)$ (Tabla II), pero resultaron mayores las puntuaciones de ganancia de cada contenido en el grupo experimental: fisiología $\left(\mathrm{PG}=5,59 ; \mathrm{t}_{(71)}=31,61\right.$; $\mathrm{p}<0,000)$, anatomía $\left(\mathrm{PG}=4,46 ; \mathrm{t}_{(71)}=27,55 ; \mathrm{p}<0,000\right)$ y salud $\left(\mathrm{PG}=2,97 ; \mathrm{t}_{(71)}=18,09 ; \mathrm{p}<, 000\right)$. Se confirma así la hipótesis del estudio. En la Figura 2 se pueden comprobar gráficamente las diferencias de mejora entre el grupo experimental y el grupo control en cada uno de los contenidos (Fig. 3). Se demuestra que corresponden al grupo que ha estudiado la teoría de las unidades didácticas mediante Moodle y Mahara las mejoras más altas respecto al pretest. Se ha alcanzado una puntuación de ganancia por encima del $55 \%$ en fisiología $(5,59)$ y del $44 \%$ en anatomía $(4,46)$ y de $29 \%$ en salud. Las mejoras en el grupo que ha estudiado mediante metodología tradicional también son destacables, pero apenas si se sobrepasan la mitad de respuestas correctas del total del test (en cada contenido), y con puntuaciones de mejora inferiores a las expuestas del grupo experimental: $37 \%$ en fisiología, $22 \%$ en anatomía y $17 \%$ en salud. 
Tabla I. Estadísticos descriptivos del total de la muestra. Medidas pretest y postest.

\begin{tabular}{lccccc}
\hline Contenidos & & $\boldsymbol{M}$ & $\boldsymbol{D T}$ & Asimetría & Curtosis \\
\hline Anatomía & Pretest & 2,78 & 0,83 & 0,08 & $-0,09$ \\
& Postest & 6,31 & 1,66 & 0,13 & $-0,80$ \\
\multirow{2}{*}{ Fisiología } & Pretest & 1,23 & 0,91 & 0,30 & $-0,69$ \\
& Postest & 6,04 & 1,41 & 0,03 & $-0,07$ \\
\multirow{2}{*}{ Salud } & Pretest & 4,30 & 1,01 & 0,16 & $-1,09$ \\
& Postest & 6,75 & 1,15 & 0,25 & $-0,35$ \\
\hline
\end{tabular}

Tabla II. Media y desviación típica de pretest, postest y puntuación de ganancia (PG). Diferencias intra-grupos. Prueba $T$ de Student para muestras relacionadas.

\begin{tabular}{lccccc}
\hline & & \multicolumn{2}{c}{ Grupo Experimental } & \multicolumn{2}{c}{ Grupo Control } \\
\cline { 3 - 6 } Contenidos & & $\boldsymbol{M}$ & $\boldsymbol{D T}$ & $\boldsymbol{M}$ & $\boldsymbol{D T}$ \\
\hline Anatomía & Pretest & 2,76 & 0,91 & 2,80 & 0,71 \\
& Postest & 7,22 & 1,37 & 5,06 & 1,14 \\
& PG & 4,46 & -- & 2,26 & --- \\
& $t$ & 27,55 & -- & 12,10 & --- \\
Fisiología & $p$ & 0,000 & -- & 0,000 & --- \\
& Pretest & 1,19 & 0,91 & 1,28 & 0,91 \\
& Postest & 6,78 & 1,12 & 5,02 & 1,09 \\
& PG & 5,59 & -- & 3,74 & --- \\
\multirow{5}{*}{ Salud } & $t$ & 31,61 & -- & 19,00 & --- \\
& $p$ & 0,000 & -- & 0,000 & --- \\
& Pretest & 4,33 & 1,08 & 4,27 & 0,91 \\
& Postest & 7,30 & 1,00 & 5,98 & 0,87 \\
& PG & 2,97 & -- & 1,71 & --- \\
& $t$ & 18,09 & -- & 9,16 & --- \\
& $p$ & 0,000 & -- & 0,000 & --- \\
\hline
\end{tabular}

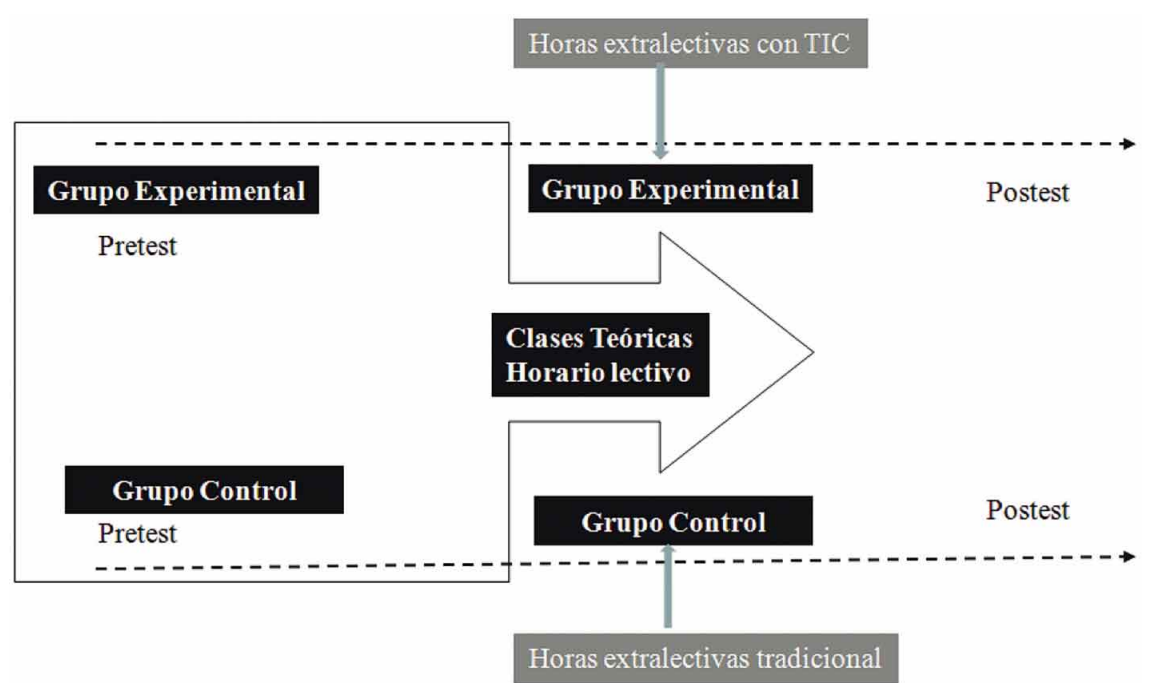

Fig. 2. Esquema del proceso realizado.

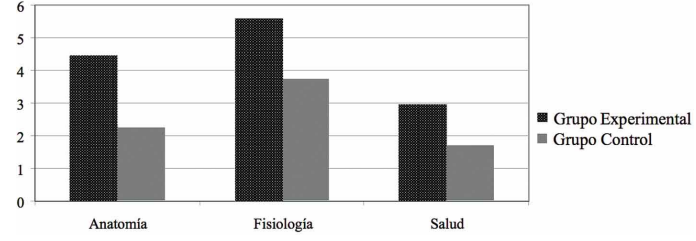

Fig. 3. Puntuaciones de ganancia del grupo experimental y del grupo control en cada contenido.

\section{DISCUSIÓN}

El objetivo de este trabajo ha sido comparar si existía mejora en el aprendizaje de los alumnos de educación secundaria tras recibir una enseñanza de contenidos de anatomía, fisiología y salud humana, con metodología tradicional o plataformas TIC. Este trabajo es importante, entre otros motivos, porque los alumnos del siglo XXI nacen con las TIC ya creadas e insertadas en su vida cotidiana (Blanco, 2013), y eso, debemos como docentes aprovecharlo, no negarnos a su uso.

Tras el proceso de intervención se ha podido comprobar cómo los mejores resultados corresponden a la salud, seguido de la anatomía y fisiología. Esto es entendible, pues dentro de los contenidos trabajados, la fisiología es reconocida por los alumnos como los contenidos más difíciles.

Pero además, con el análisis estadístico se ha podido observar que tras la intervención se han producido mejoras significativas tanto en el grupo experimental como en el grupo control, siendo mayores las puntuaciones de ganancia de fisiología, anatomía y salud en el grupo experimental.

Estos datos corroboran que la utilización de las plataformas LMS (Moodle 2.0 y Mahara integrado) contribuyen en mayor medida a la adquisición de estos contenidos entre el alumnado. Pero en cambio, según Gutiérrez et al., las TIC no son siempre beneficiosas en el proceso de enseñanza-aprendizaje, ya que su supuesta "bondad" en todos los campos, incluida la educación, es parte, según estos autores, de su estrategia de mercado que no siempre es buena en educación. 
Siguiendo a Ardura \& Zamora (2014) y Domínguez (2010), se ha podido comprobar como Moodle 2.0 y Mahara ya no son sólo unas herramientas propias de la educación a distancia, sino que son un complemento de uso muy útil en la enseñanza presencial, pues los resultados de esta investigación así lo demuestran. Estas plataformas ayudan en el aprendizaje de contenidos de fisiología y anatomía que por lo general son muy complejos de aprender para los alumnos. De hecho, estas aplicaciones podrían solventar algunos de los problemas planteados en estas enseñanzas, como la obtención, conservación, calidad y cantidad de los elementos anatómicos a estudiar con el alumnado (Da Silva et al., 2013). Por ello es fundamental trabajar con estos recursos que permitan apoyar las clases teóricas y con tareas que permitan progresar al ritmo de los discentes. Por tanto, estamos de acuerdo con Domínguez al afirmar que gracias a esta plataforma también pueden gozar los alumnos de mayor autonomía en cuanto a ritmo de aprendizaje y estudio (algo que motivará al alumnado en su aprendizaje, según Baena-Extremera et al. (2013) lo que ha supuesto que haya un mayor aprendizaje en aquellos contenidos más difíciles. Igualmente Betegón et al. utilizan la plataforma Moodle como apoyo a la docencia presencial en sus clases. Los resultados del presente trabajo ratificando las conclusiones de estos autores.

Otra de las grandes diferencias entre el grupo experimental y el grupo control puede ser debida al incremento del nivel de motivación de los discentes hacia los contenidos impartidos en clase a través de las TIC. De hecho, autores como Domínguez creen que el uso de las TIC en la enseñanza hoy día es en todos los niveles, una manera de atraer y captar la atención del alumnado, mejorando por tanto las posibilidades de aprendizaje.

Coincidiendo con Ros (2008), otra ventaja observada en esta investigación en el grupo experimental es que el uso de estas plataformas permite la integración de diferentes alumnos con discapacidades físicas, sensoriales o psíquicas, ya que todos los recursos que usemos, pueden ser adaptados (videos con subtítulos, videos narrados, etc.). Por ello, al igual que ocurre con el trabajo de Prieto et al., la utilización de Moodle 2.0 y Mahara optimiza el trabajo individual y ayuda a atender a la diversidad de los alumnos.

En la línea descrita por Domínguez se ha podido comprobar cómo la mejora en el grupo experimental es posiblemente debida también a que el uso de estas nuevas plataformas de aprendizaje permiten al profesorado la posibilidad de ofrecer mayor variedad de materiales para el aprendizaje de un tema, tanto teóricos, como audiovisuales, referencias bibliográficas o a páginas webs educativas relacionadas con la materia, la creación de foros, aulas virtuales, etc. Autores como Agüera \& de la Haba utilizan la plataforma Moodle para subir diverso material audiovisual realizado durante los trabajos de laboratorio. Así, según estos autores, este tipo de soportes digitales multiplataforma aportan al alumno mucha información visual que, en la manera tradicional de impartir las prácticas no observan y tienen que deducirla a partir de la explicación del profesor.

Una importante aportación en el campo de la EF lo constituye el hecho de que sean necesarias menos horas presenciales de carácter teórico, pues el alumnado trabaja en horario extralectivo sobre los contenidos prácticos realizados en clase, disponiendo así de más horas de práctica lectiva. A este incremento de horas para la práctica hay que añadir, dados los datos de este estudio, que el alumnado asimile mejor y de forma más significativa los contenidos teóricos planteados. Se puede concluir que el uso de estas plataformas de aprendizaje mejora notablemente los resultados en el alumnado tras los efectos de la intervención. Pero, siguiendo a Prieto et al., entendemos que las TIC pueden servir de apoyo al trabajo en el aula, pero no reemplazan, en ningún caso, al profesor.

\section{AGRADECIMIENTOS}

Los autores agradecen la colaboración del IES Cardenal Cisneros de Albox (Almería), así como el apoyo para el uso del aula virtual del Centro del Profesorado CuevasOlula (Almería).

GRANERO-GALLEGOS, A. \& BAENA-EXTREMERA, A. Patterns of ICT-based learning (Moodle and Mahara 2.0) for contents of anatomy, physiology and health in scholar Physical Education lessons. Int. J. Morphol., 33(1):375-381, 2015.

SUMMARY: The aim of this study was to compare the learning performance of students in secondary physical education lessons after receiving two teaching units in which the theoretical content of Anatomy, Physiology and Body Health, was given with the support of Moodle and Mahara 2.0. We used a quasi-experimental, descriptive and sectional design. The total sample consisted of 122 students in the 4th year of Secondary Education (ESO), 57 males and 65 females. We worked with an experimental group of 72 people who received theoretical training of two teaching units supported by Moodle 2.0 and Mahara, and a control group of 50 students who received no support classes of virtual platforms. Practical classes were similar in both groups. The measuring instrument was a multiple choice assessment questionnaire. Pretest measure was obtained before training and other measures after the intervention (posttest). Improvements were analyzed in each group according to the theoretical method used and compared the results obtained by analysis of gain score (posttest-pretest) with T-test for related samples. After intervention, the average score is greater than fifty percent in both groups, without significant gain scores of physiology, anatomy and health in the experimental group. These data confirm that the use of LMS platforms contribute more to the acquisition of this content among students. An important contribution in the field of EF is the fact that less theoretical contact hours are 
necessary, as the students work on time on the practical elective content made in class, thus having more hours of practice teaching time.

KEY WORDS: Methodology; Moodle 2.0; Mahara; Anatomy; Physiology; Physical education.

\section{REFERENCIAS BIBLIOGRÁFICAS}

Agüera, B. E. \& de la Haba, H. P. Desarrollo de nuevas tecnologías de la información y la comunicación (TIC) para la docencia práctica en el área de conocimiento de fisiología vegetal. Educar, 44:59-65, 2009.

Algieri, R. D.; Mazzoglio y Nabar, M. J.; Dogliotti, C. G. \& Gazzotti, A. TICs Applied to Teaching the Digestive Apparatus. Int. J. Morphol, 27(4):1261-8, 2009

Alleyne, T.; Shirley, A.; Bennett, C.; Addae, J.; Walrond, E.; West, S. \& Pinto Pereira, L. Problem-based compared with traditional methods at the Faculty of Medical Sciences, University of the West Indies: a model study. Med. Teach., 24(3):273-9, 2002.

Ardura, D. \& Zamora, A. ¿Son útiles los entornos virtuales de aprendizaje? Evaluación de una experiencia en la enseñanza y el aprendizaje de la Relatividad. Rev. Eureka Enseñ. y Divulg. Cienc., 11(1):83-93, 2014.

Baena-Extremera, A. \& Granero-Gallegos, A. Using iBook in Teaching Anatomy Content in Secundary Education. Int. J. Morphol., 31(2):50511, 2013.

Baena-Extremera, A.; Granero-Gallegos, A.; Sánchez-Fuentes, J. A. \& Martínez-Molina, M. Apoyo a la autonomía en Educación Física: antecedentes, diseño, metodología y análisis de la relación con la motivación en estudiantes adolescentes. Retos, (24):46-9, 2013.

Blanco, E. A. El uso y el conocimiento de los contenidos de educación física en Internet del alumnado de segundo ciclo de Educación Secundaria Obligatoria. Estudio de caso. Enseñ. Teach., 31(2):69-92, 2013.

Best, J. W. Research in education. Englewood Cliffs N. J., Prentice Hall, 1970.

Betegón, S. L.; Fossas, O. M.; Martínez, R. E. \& Ramos, G. M. M. Entornos virtuales como apoyo a la docencia universitaria presencial. Utilidad de Moodle. Anu. Juríd. Econ. Escur., (43):273-302, 2010.

Bollen, K. A. \& Long, J. S. Testing structural equation models. Newbury Park C. A., Sage, 1994.

Bucarey A., S.; Araya A., E.; Cabezas O., X. \& Álvarez G., L. Anatomy Contents in Learning Designs Displayed in LAMS and Integrated to Moodle. Int. J. Morphol., 29(2):363-70, 2011

Correa, J. M. \& de Pablos, J. Nuevas tecnologías e innovación educativa. Rev. Psicodidáct., 14(1):133-45, 2009.

Da Silva, K. D. C.; Santana, O. A. \& De Moraes, S. R. A. Quality and language of learning objects used in the teaching of human anatomy. Int. J. Morphol, 31(2):455-60, 2013.

Domínguez, L. M. R. Moodle, una plataforma formativa con gran proyección en los nuevos modelos de enseñanza. Rev. Didact. Innov. Multimed., 19,: 2010.
Granero-Gallegos, A. Una experiencia de teleformación del profesorado de Educación Física (Primaria y Secundaria) en actividades físicas en el medio natural. Retos, (13):39-45, 2008.

Gutiérrez, M. A.; Palacios, P. A. \& Torrego, E. L. La formación de los futuros maestros y la integración de las TIC en la educación: anatomía de un desencuentro. Rev. Educ., (352):1-17, 2010.

Jones, A.; McArdle, P. J. \& O’Neill, P. A. Perceptions of how well graduates are prepared for the role of pre-registration house officer: a comparison of outcomes from a traditional and an integrated PBL curriculum. Med. Educ., 36(1):16-25, 2002.

Padilla-Meléndez, A.; Del Águila-Obra, A. R. \& Garrido-Moreno, A. Empleo de moodle en los procesos de enseñanza-aprendizaje de dirección de empresas: nuevo perfil del estudiante en el EEES. Educ. XX1, 18(1):125-46, 2015.

Pérez, A. Un paseo por Moodle. Prim. Not. Comun. Pedag., (210):69-71, 2006.

Potu, B. K.; Shwe, W. H.; Jagadeesan, S.; Aung, T. \& Cheng, P. S. Scope of Anatomy Teaching in Problem-based Learning (PBL) Sessions of Integrated Medical Curriculum. Int. J. Morphol., 31(3):899-901, 2013.

Prieto, R.; Smok, C. \& Rojas, M. Blog Experiences: Compared Placenta. Int. J. Morphol., 29(2):432-5, 2011

Agencia Estatal Boletín Oficial del Estado. Real Decreto 1631/2006 de 29 de diciembre, por el que se establecen las enseñanzas mínimas para la Educación Secundaria Obligatoria en España, para el área de Educación Física. Madrid, Ministerio de la Presidencia, Gobierno de España, 2006. Disponible en: http://www.boe.es/diario_boe/ txt.php?id=BOE-A-2007-238

Ramos, A. M. J. \& Martínez, L. F. J. Estrategias y metodología frente al desarrollo de una acción formativa virtual. Una propuesta práctica con alumnado en educación secundaria. Espiral, 4(8):32-41, 2011.

Rodríguez, R. S. El impacto de las TIC en la transformación de la enseñanza universitaria: repensar los modelos de enseñanza y aprendizaje. Teor. Educ. Educ. Cult. Soc. Inf., 11(1):32-68, 2010.

Ros, I. Moodle, la plataforma para la enseñanza y organización escolar. Ikastorratza, e-Revista de didáctica, 2:1-12, 2008. Disponible en: http:/ /www.ehu.eus/ikastorratza/2_alea/moodle.pdf

Suárez-Rodríguez, J. M.; Almerich, G.; Gargallo López, B. \& Aliaga, F. M. Las competencias del profesorado en TIC: estructura básica. Educ. XX1, 16(1):39-62, 2013

\section{Dirección para Correspondencia: \\ Dr. Antonio Baena Extremera \\ Facultad de Ciencias del Deporte \\ Universidad de Murcia \\ C/ Argentina s/n Santiago de la Ribera \\ Murcia 30720 \\ ESPAÑA}

Email: abaenaextrem@um.es

Recibido : 11-02-2012

Aceptado: 17-11-2014 